\title{
Study on the Synergetic Mechanism for the Dynamic Evaluation of Electricity Market Operational Efficiency
}

\author{
Chunjie Li, Li Yan, Huiru Zhao \\ North China Electric Power University, Beijing, China \\ E-mail: houlaiyanli@126.com \\ Received May 10, 2011; revised May 20, 2011; accepted June 7, 2011
}

\begin{abstract}
In Synergetics, when a complex system evolves from one sate to another, the order parameter plays a dominant role. We can analyze the complex system state by studying the dynamic of order parameter. We developed a synergetic model of electricity market operation system, and studied the dynamic process of the system with empirical example, which revealed the internal mechanism of the system evolution. In order to verify the accuracy of the synergetic model, fourth-order Runge-Kutta algorithm and grey relevance method were used. Finally, we found that the reserve rate of generation was the order parameter of the system. Then we can use the principle of Synergetics to evaluate the efficiency of electricity market operation.
\end{abstract}

Keywords: Electricity Market Operation, Synergetics, Synergetic Model, Order Parameter, Runge-Kutta Algorithm, Grey Relevance Method

\section{Introduction}

The electricity market operation, involving the technical and economic relations between generating, transmitting, distributing and retailing parts which must be balanceable at anytime, is a complex system that is open, nonlinear and dynamic. The efficiency evaluation of electricity market operation system (EMOS) cannot simply apply the traditional method of the input-output principle, but should be in the point of overall dynamic operation. Based on the synergetic theory, we constructed a synergetic model and found the order parameter of EMOS. By studying the change of the order parameter which reflected the system operation state, the efficiency evaluation mechanism of electricity market operation was revealed.

\section{Introduction of Synergetics}

Synergetics was created by Haken, a German physicist. It is a self-organized theory studying nonlinear interaction between subsystems which will result in ordered evolution of the system structure. The key point of Synergetics is how an open, erratic system evolves from a disordered structure to an ordered state, or from one ordered state to another one.

The general steps dealing with the issues of Synergetics are as following $[1,2]$ :
1) "Translate" the specific issues to mathematical problems. Explore the relationships between key variables and constants which these two parameters determine the system operation state. Then construct mathematical model. The basic evolution equations of Synergetics can be written in the form of Langevin equations:

$$
\dot{u}=K(u, s)+F
$$

$\dot{u}$ is the change rate of state variables; $u$ are state variables that can be macro or micro; $s$ are control parameters; $F$ is random noise. The evolution model is differential equation or equations containing $u$ and its derivative.

2) In order to test the accuracy of the synergetic model, calculate with computer. Then compare the simulated results with initial values.

3) Find order parameters in the model by distinguishing the size of damping coefficients.

4) Based on the Synergetic servo principle, cancel fast variables and get order parameter equations. By analyzing the dynamic of the order parameter, evaluate the state of the system.

\section{Synergetic Features of Electricity Market Operation}

Synergetics is mainly used to study open, non-linear, 
erratic complex systems. The open feature means that the system exchanges energy, material and information with outside. The non-linear feature shows that the nonlinear mechanism between variables of the system exists. The essence of unbalance is that the system state is changing with time [3].

EMOS is an open system that exchanges energy, material and information with natural and social environment. For example, coal-fired power plants need to purchase coal in the energy market. The construction investment of the plants is affected by national economic development and macroeconomic policy, etc.

Although there are complex technical and economic relations between the generating, transmitting, distributing and retailing subsystems, they must be coordinate highly, e.g. the variables of EMOS varied with time, but they are generally in coordination and non-linear conditions.

EMOS is in erratic state. For reasons above, in the process of electricity market operation, the system exchanges material and information with outside, and provides power to users by tracking load at any time. In other word, the system is always in dynamic equilibrium state [4].

For all above, EMOS has synergetic evolution features of complex system and accords with the principles of Synergetics.

\section{Synergetic Model of Electricity Market Operation}

\subsection{Select State Variables}

To create the synergetic model of EMOS, we should select the state variables first.

Based on the SCP principle of Harvard School, the electricity market is divided into three parts: market structure, market conduct and market performance. In SCP, structure determines conduct, further more, conduct determines performance. But it's not a simple one-way relationship. At the same time, conduct can affect structure, and performance can react on structure and conduct either [5-7]. For above, we divide the EMOS into three subsystems: structure subsystem, conduct subsystem and performance subsystem. Four state variables are selected: declared supply and demand ratio (SDR), reserve rate of generation (RG), transacted power amount (TP) and market clearing price (CP). These state variables are described in Table 1.

\subsection{Synergetic Model}

Let $X_{1}(t), X_{2}(t), X_{3}(t), X_{4}(t)$ represent SDR, RG,
Table 1. Description of the state variables of electricity market operation.

\begin{tabular}{cl}
\hline $\begin{array}{c}\text { State } \\
\text { variables }\end{array}$ & \multicolumn{1}{c}{ Implication } \\
\hline SDR & The formula is: declared supply/declared demand. \\
RG & $\begin{array}{l}\text { The formula is: (available generation amount-actual } \\
\text { power demand)/available generation amount }\end{array}$ \\
TP & The actual transacted power amount after bidding. \\
CP & The market clearing price after bidding. \\
\hline
\end{tabular}

TP and CP individually. Based on Synergetics, we suppose that the change rate of $X_{i}(t)$ is $\mathrm{d} X_{i}(t) / \mathrm{d} t$ which is determined by following: the first factor is the inside synergy effect of the state variables. That is the development and inhibition effect of the state variable itself. The other factor is the outside synergy effect of the state variables. That is the cooperation and competition effect from the other state variables. The inside synergy items of the $i$ th state variable are $a_{i i} X_{i}(t)$ and $b_{i i} X_{i}^{2}(t)$. The outside synergy items are $a_{i j} X_{j}(t)$ and $b_{i j} X_{j}^{2}(t)$. We cannot tell which ones are development and cooperation items, and which are inhibition and competition ones. So it is assumed that the coefficients of all items are positive. The exact symbol will be given by the model results.

Based on the discussion above, the synergetic model of EMOS is:

$$
\begin{aligned}
\frac{\mathrm{d} X_{i}(t)}{\mathrm{d} t}= & a_{i i} X_{i}(t)+b_{i i} X_{i}^{2}(t) \\
& +\sum_{j=1, j \neq i}^{4}\left(a_{i j} X_{j}(t)+b_{i j} X_{j}^{2}(t)\right)+f_{i}(t)
\end{aligned}
$$

In model (1), $f_{i}(t)$ is the outside interference that is influenced by environment and policy. The main purpose of this paper is to find the order parameter of EMOS by studying the interaction of state variables, but not to forecast when the electricity market develops from an ordered state to another by the help of outside interference. So we suppose $f_{i}(t)=0$ in this paper.

\subsection{Find the Order Parameters}

In Synergetics, when system tends to the critical point, it evolves from a disordered structure to an ordered state, or from one ordered state to another one. At this point, the state variables will develop into two categories: variables changing rapidly with time are known as the fast variables; the other kinds change very slowly, known as the slow variables which are the order parameters. The order parameter, a core concept of Synergetics, can lead to new structure of the system, and reflect the ordered 
degree of the new structure [8-11]. Haken established two synergetic research methods: micro and macro method. From the point of micro method, we can find the order parameters through the size of damping coefficients in synergetic model. And from the point of macro method, the principle of maximum information entropy is used to find the order parameters. But some problems exit in macro method: it is not sure that the information entropy increases or deceases when the system is developing from one state to another. So we use the micro method to find order parameter in this paper.

\section{Case Study}

\subsection{The Synergetic Model of a Regional Electricity Market}

Based on the synergetic model of EMOS above, we will analyze a regional electricity market in this part. Table 2 is the operation data of the market in year 2008 and 2009.

Since the unit and magnitude of the initial data is different, we normalize the data first:

$$
\begin{aligned}
& X_{i}^{(0)}(t)=\frac{X_{i}(t)-\min _{t} X_{i}(t)}{\max _{t} X_{i}(t)-\min _{t} X_{i}(t)} \\
& (i=1,2, \cdots, 4 ; t=1,2, \cdots 24) .
\end{aligned}
$$

In order to weaken the randomness of the time series, we cumulate the normalized data, and get new series $X_{i}^{(1)}(t)$ :

$$
X_{i}^{(1)}(k)=\sum_{t=1}^{k} X_{i}^{(0)}(t), \quad(k=1,2, \cdots 24) .
$$

Using least square method, we substitute the data above into the synergetic model (1), and get the equations with the help of MATLAB: (see (2))

This is the synergetic model of the regional electricity market.

\subsection{Verifying the Model}

In model (2), in order to simplify the calculation, the first and second power of the items were considered, the higher power items were omitted. So the accuracy of the synergetic model needs to be verified, we use fourthorder Runge-Kutta method and grey relevance method to verify the model.

\subsubsection{Verifying by Fourth-Order Runge-Kutta Algorithm}

Using fourth-order Runge-Kutta algorithm, we got the numerical solution of model (2). Comparing the simulated data $\tilde{X}_{i}^{(1)}(t)$ with initial data $X_{i}^{(1)}(t)$, we made the trend figures of these data. See Figure 1 and Figure 2.

We can see the trends of initial data and simulated data are identical approximately. So model (2) is reasonable.

\subsubsection{Verifying by Grey Relevance Method}

The fit degree of the model has been displayed intuitively above. To verify the model further, we calculate the grey relevance degree of the initial and simulated data series $[12,13]$. The result is in Table 3.

Based on the grey relevance theory, the relevance coefficient is 0.5 generally. If the grey relevance degree is more than 0.6, we can consider the model is satisfied. From Table 3, the results are all more than 0.6. So model (2) is good.

$$
\left\{\begin{aligned}
\frac{\mathrm{d} X_{1}^{(1)}(t)}{\mathrm{d} t}= & 0.1971 X_{1}^{(1)}(t)-0.0244\left(X_{1}^{(1)}(t)\right)^{2}+0.3573 X_{2}^{(1)}(t)+0.4643 X_{3}^{(1)}(t)-0.2675 X_{4}^{(1)}(t) \\
& -0.0252\left(X_{2}^{(1)}(t)\right)^{2}+0.0096\left(X_{3}^{(1)}(t)\right)^{2}-0.0158\left(X_{4}^{(1)}(t)\right)^{2} \\
\frac{\mathrm{d} X_{2}^{(1)}(t)}{\mathrm{d} t}= & -0.0906 X_{2}^{(1)}(t)-0.0016\left(X_{2}^{(1)}(t)\right)^{2}-0.7349 X_{1}^{(1)}(t)+0.0141 X_{3}^{(1)}(t)+0.7306 X_{4}^{(1)}(t) \\
& +0.0607\left(X_{1}^{(1)}(t)\right)^{2}+0.0051\left(X_{3}^{(1)}(t)\right)^{2}-0.0421\left(X_{4}^{(1)}(t)\right)^{2} \\
\frac{\mathrm{d} X_{3}^{(1)}(t)}{\mathrm{d} t}= & -0.2818 X_{3}^{(1)}(t)-0.0151\left(X_{3}^{(1)}(t)\right)^{2}-0.0237 X_{1}^{(1)}(t)-0.0555 X_{2}^{(1)}(t)+0.4852 X_{4}^{(1)}(t) \\
& -0.0333\left(X_{1}^{(1)}(t)\right)^{2}+0.0582\left(X_{2}^{(1)}(t)\right)^{2}-0.0281\left(X_{4}^{(1)}(t)\right)^{2} \\
\frac{\mathrm{d} X_{4}^{(1)}(t)}{\mathrm{d} t}= & -0.3059 X_{4}^{(1)}(t)-0.0099\left(X_{4}^{(1)}(t)\right)^{2}+0.5345 X_{1}^{(1)}(t)-0.0397 X_{2}^{(1)}(t)+0.6089 X_{3}^{(1)}(t) \\
& -0.0267\left(X_{1}^{(1)}(t)\right)^{2}+0.0083\left(X_{2}^{(1)}(t)\right)^{2}-0.043\left(X_{3}^{(1)}(t)\right)^{2}
\end{aligned}\right.
$$


Table 2. Initial data of the state variables of a regional electricity market.

\begin{tabular}{|c|c|c|c|c|}
\hline Time & SDR & RG & TP (MWH) & CP (Dollar/MWH) \\
\hline 200801 & 1.00211 & $20.747 \%$ & $35,233.22$ & 65.85938 \\
\hline 200802 & 0.989038 & $17.101 \%$ & $34,450.82$ & 65.17588 \\
\hline 200803 & 0.987977 & $15.667 \%$ & $31,492.05$ & 67.56227 \\
\hline 200804 & 0.978164 & $16.257 \%$ & $28,719.23$ & 69.98486 \\
\hline 200805 & 0.983068 & $17.424 \%$ & $28,443.79$ & 60.5116 \\
\hline 200806 & 0.968073 & $20.908 \%$ & $36,217.86$ & 70.27707 \\
\hline 200807 & 0.970237 & $21.047 \%$ & $38,767.71$ & 69.57751 \\
\hline 200808 & 0.974491 & $22.615 \%$ & $34,377.53$ & 67.63856 \\
\hline 200809 & 1.001009 & $22.549 \%$ & $32,799.74$ & 61.22847 \\
\hline 200810 & 0.992952 & $19.438 \%$ & $29,102.55$ & 58.78035 \\
\hline 200811 & 0.972513 & $21.081 \%$ & $30,317.73$ & 51.80096 \\
\hline 200812 & 0.96716 & $18.899 \%$ & $32,860.43$ & 49.01535 \\
\hline 200901 & 0.959662 & $16.915 \%$ & $35,209.08$ & 59.18817 \\
\hline 200902 & 0.966923 & $16.978 \%$ & $32,796.22$ & 43.94823 \\
\hline 200903 & 0.963481 & $18.017 \%$ & 29,985.82 & 39.60535 \\
\hline 200904 & 0.98675 & $17.786 \%$ & $28,881.85$ & 33.40889 \\
\hline 200905 & 0.95921 & $19.097 \%$ & $27,470.32$ & 32.27513 \\
\hline 200906 & 0.957677 & $19.510 \%$ & $31,276.32$ & 33.0665 \\
\hline 200907 & 0.962913 & $17.554 \%$ & $33,672.87$ & 32.12929 \\
\hline 200908 & 0.976382 & $17.766 \%$ & $37,328.11$ & 34.35247 \\
\hline 200909 & 0.976117 & $18.205 \%$ & $29,548.8$ & 29.71408 \\
\hline 200910 & 1.002916 & $19.085 \%$ & $28,641.84$ & 33.75836 \\
\hline 200911 & 0.976794 & $19.657 \%$ & $28,550.08$ & 32.33904 \\
\hline 200912 & 0.974989 & $22.452 \%$ & $33,400.78$ & 42.03409 \\
\hline
\end{tabular}

Data source: www.pjm.com.

Table 3. The grey relevance degree of initial and simulated data of state variables.

\begin{tabular}{ccccc}
\hline & $\gamma\left(X_{1}^{(1)}(t), \tilde{X}_{1}^{(1)}(t)\right)$ & $\gamma\left(X_{2}^{(1)}(t), \tilde{X}_{2}^{(1)}(t)\right)$ & $\gamma\left(X_{3}^{(1)}(t), \tilde{X}_{3}^{(1)}(t)\right)$ & $\gamma\left(X_{4}^{(1)}(t), \tilde{X}_{4}^{(1)}(t)\right)$ \\
\hline Grey relevance degree & 0.665 & 0.623 & 0.606 & 0.708
\end{tabular}

\subsection{The Order Parameter of the Regional Electricity Market}

From synergetic model of EMOS (2), the damping coefficients are $a_{11}=0.1971, a_{22}=-0.0906, a_{33}=-0.2818$, $a_{44}=-0.3059$. Based on Synergetics, the state variable of which damping coefficient is the least is the order parameter. Since the damping coefficient of $X_{2}(t)$ is the least, i.e. RG is the order parameter of the regional electricity market. As an index reflecting market structure, RG reflects the match degree of generation capacity and market demand. In other word, RG which reflects 


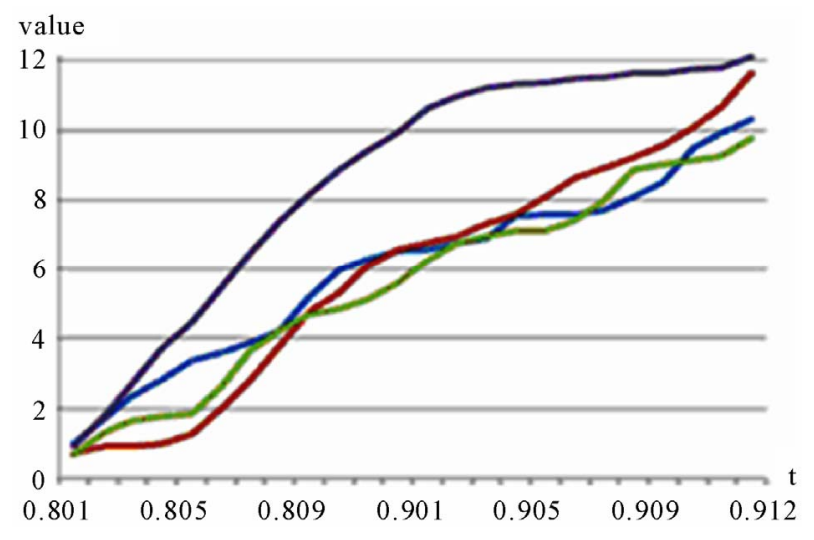

Figure 1. The trend of initial data.

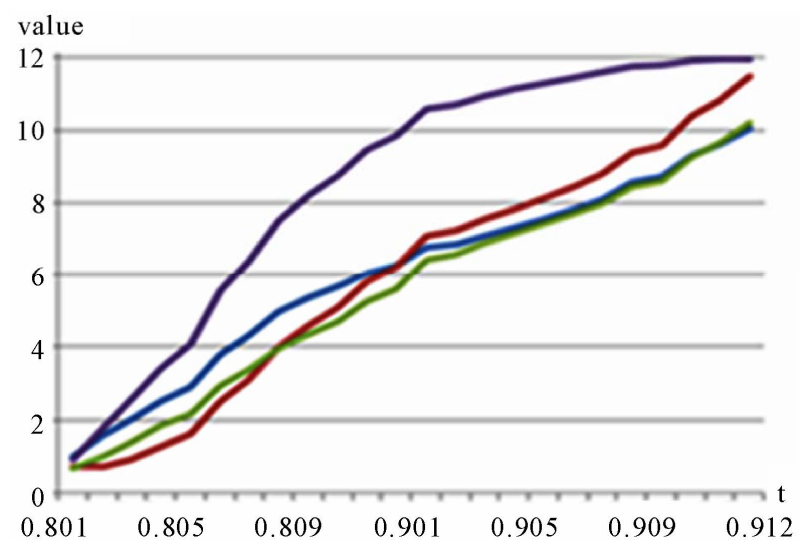

Figure 2. The trend of simulated data.

the situation of supply and demand in the electricity market implies whether the market power exits.

\section{Conclusions}

The electricity market operation system which is open, non-linear and erratic, achieves to ordered operation state through continuous development. Therefore, the evolution of EMOS accords with Synergetics theory. Based on Synergetics, we divided EMOS into structure, conduct and performance subsystems. Then we selected several state variables and constructed synergetic model of EMOS. Through empirical analysis, reserve rate of generation was proofed the order parameter. Based on this paper, we can track the dynamic state of electricity market operation by studying the change of order parameter, and give the mechanism for the efficiency evaluation of electricity market operation.

\section{References}

[1] Z. H. Song, “A Study on Integrated Mechanism of Metallurgy Enterprise Technology Innovation Based on Synergetic Theory,” Tianjin University, Tianjin, 2006.

[2] T. Wu, "Theory of Cooperative Methods-Self-Organization and Its Application,” Inner Mongolia Social Science, Vol. 11, No. 6, 2000, pp. 19-26.

[3] H. Hermann, “Advanced Synergetics,” Beijing Science Press, Beijing, 1989.

[4] H. Hermann, "Information and Self-Organization,” Sichuan Education Press, Chengdu, 1988.

[5] Q. Wang, F. S. Wen and M. Liu, "Development of Evaluation Indices System for Power Market," Electric Power Technology Economics, Vol. 20, No. 5, 2008, pp. 21-26.

[6] H. F. Li, "Research of the Evaluation Index System and the Evaluation Model about Electric power Market in China,” North China Electric Power University, Beijing, 2008.

[7] D. N. Liu, R. Q. Li and X. Q. Chen, "Regulatory Indicators and Market Assessment System of Electricity Market," Automation of Electric Power Systems, Vol. 28, No. 9, 2004, pp. 16-21.

[8] L. Jin, "Research on the Construction System Evolutionary Development Based on Self-Organization Theory,” Harbin Institute of Technology, Harbin, 2007.

[9] P. Y. Zhang and C. J. Li, "Study on Operational Efficiency of Electricity Market Based on Synergetic Theory," The International Conference on Electrical and Control Engineering, Wuhan, 25-27 June 2010, pp. 281-284. doi:10.1109/iCECE.2010.75

[10] J. B. Liu and B. Z. Li, "Study on the Order Parameters of the Enterprise Evolution System,” China Science and Technology Forum, Vol. 7, No. 4, 2005, pp. 85-87.

[11] J. Z. Wang, “Technology Self-Organization Evolution Mechanism and the Order Parameter," Dialectics of $\mathrm{Na}$ ture, Vol. 23, No. 4, 2007, pp. 47-49.

[12] C. Cheng and C. J. Li, “Application of Grey Incidence Analysis in the Regional Electricity Market Efficiency Evaluation," Journal of North China Electric Power University, Vol. 6, 2010, pp. 10-13.

[13] S. Y. Wei and J. Y. Wu, "Short-Term Bus Load Forecasting Based on Grey Model and Kalman Smoother," Transactions of China Electrotechnical Society, Vol. 25, No. 2, 2010, pp. 158-162. 